\title{
Steady-State Fuzzy Modeling of Ultrasonic Motor System
}

\author{
Shi Jingzhuo and Lv Lin \\ Electronic Information Engineering College, Henan University of Science and Technology, Luoyang 471000, China
}

Correspondence should be addressed to Shi Jingzhuo, sjznew@163.com

Received 24 March 2011; Revised 6 September 2011; Accepted 6 September 2011

Academic Editor: Agostino Bruzzone

Copyright ( $\odot 2011$ S. Jingzhuo and L. Lin. This is an open access article distributed under the Creative Commons Attribution License, which permits unrestricted use, distribution, and reproduction in any medium, provided the original work is properly cited.

\begin{abstract}
Because of the complicated energy conversion process, ultrasonic motor has remarkable nonlinearity. Therefore, it is difficult to achieve accurate speed control of ultrasonic motor. In general, a good model is the foundation of the accurate control effect. Because of the remarkable non-linearity of ultrasonic motor, the model should also be nonlinear. In this paper, the two-input and one-output steady-state model of ultrasonic motor system is worked out using fuzzy reasoning modeling method. Comparison between experimental data and calculated data of the model prove that the model can well simulate the nonlinear relationship among the amplitude of driving voltage, frequency, and rotating speed.
\end{abstract}

\section{Introduction}

In classical and modern control theories, analyzing and designing is based on the model of controlled object. The same applies to the motion control system of ultrasonic motor, establishing an appropriate model that can obtain favorable control performance. The model can be a mathematical model based on theoretical analysis and also can be in other proper forms.

On one hand, ultrasonic motor have no accurate theoretic model, because the process of electromechanical energy conversion, which contains piezoelectric energy conversion and friction conversion, has nonlinear and dispersion characteristics [1-3]. On the other hand, ultrasonic motor does not have a long history. In order to design a controller and increase the online control performance, the model of ultrasonic motor system for control application gets more and more attention [4-11]. Generally, for the purpose of online realization and minimizing the amount of online calculation, the control model should be relatively simple and can show the main aspects of nonlinear control of ultrasonic motor system $[6,7]$. Control modeling usually adopts identification method based on the tested data, and the forms of model can be the transfer function [8], differential equation, neural network [9-11], and so forth. Among them, neural network contains nonlinear information, so it is usually used to establish the nonlinear model of ultrasonic motor system.
In recent years, fuzzy modeling method based on fuzzy reasoning is gradually arising [6]. The same as the neural network model, a fuzzy model is also easy to show the nonlinear information. It is different in that fuzzy reasoning simulates people's fuzzy thinking process. So a fuzzy model is more easily mixed and makes use of people's relevant experience knowledge. Another effective way is provided for the modeling of nonlinear complex system.

The Fuzzy Modeling Method is Rarely Used in The Field of Motor. In This Paper, The Method is Used to Establish The Model of Ultrasonic Motor System. Based on The Experimental Data, The Two-Input And One-Output Steady-State Fuzzy Model of Ultrasonic Motor System is Worked Out, And The model can well show the nonlinear dynamic relationship among the amplitude of driving voltage, frequency, and rotating speed.

\section{Experimental Data Used for Modeling}

The block diagram of experimental system is shown in Figure 1. The traveling ultrasonic motor used here is the commercial ultrasonic motor, Shinsei USR60. Phase-shift PWM method is used in the circuit to control the $\mathrm{H}$-bridge driver. Supply voltage of the circuit is DC12V. The control part is made up of DSP and CPLD. In the control circuit, DSP is used to implement the control strategies. It contains voltage controllers which are used to control the driving 


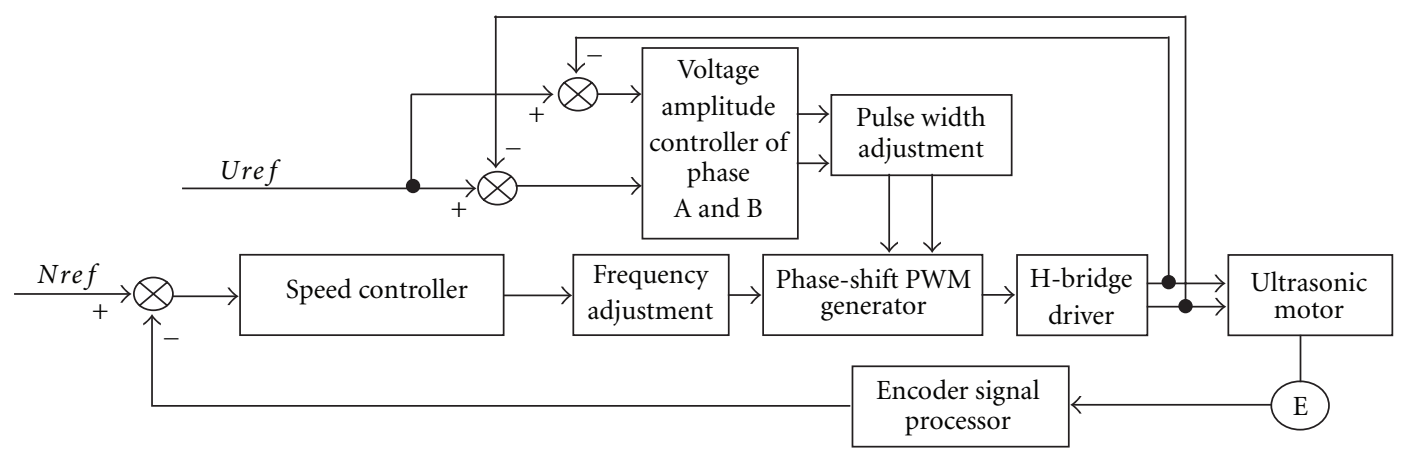

FIgURE 1: Structure of the experimental system for speed control.

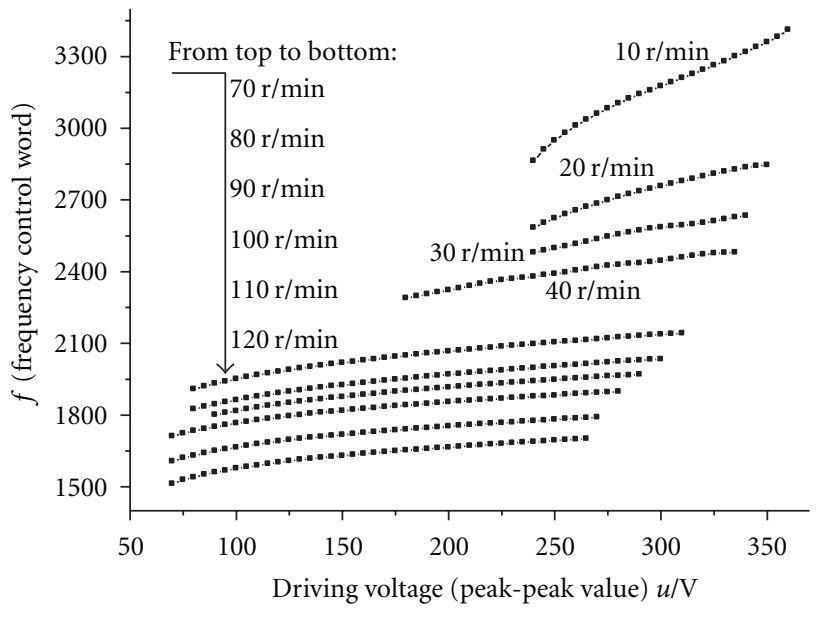

Figure 2: Tested data of speed control.

voltage amplitude of phases $\mathrm{A}$ and $\mathrm{B}$, and rotating speed controller which is used to implement rotating speed control by adjusting driving frequency. In Figure 1, Nref and Uref are the given values of rotating speed and driving voltage amplitude, respectively.

The steady-state relation data among the amplitude of driving voltage, frequency, and rotating speed are obtained as follows. Nref is set to a given value, and the driving voltage amplitude of phases A and B is changed by adjusting Uref gradually. With the change of voltage amplitude, the frequency will change in order to maintain the rotating speed at Nref by speed closed loop controller. When the adjustment reaches the steady-state condition, corresponding to different values of Uref, the value of frequency and rotating speed are recorded. And then, Nref is changed in the range of rotating speed, and the above processes are repeated. The tested data can be obtained as shown in Figure 2. In this figure, black diamonds represent experiment data, abscissa represents the driving voltage amplitude (peak-peak value), and ordinate represents the frequency control word which has a linear relationship with frequency value. The frequency control word is a description form about frequency in the control program of DSP. The frequency control word is used to establish the model for convenient to online control application. In Figure 2, every curve represents data which have the same rotating speed value. Rotating speed rises from top to bottom, and the values are marked in Figure 2. When the value of rotating speed is different, the range of driving voltage amplitude of ultrasonic motor is also different in natural running. So the lengths of curves are different in Figure 2.

\section{The Steps of Fuzzy Modeling}

The basic structure of fuzzy model is shown in Figure 3. The model includes input quantization, fuzzification (calculating the degree of membership), fuzzy reasoning based on fuzzy rules, defuzzification, and output proportional computing. The steps of establishing a fuzzy model are as follows.

(1) Divide the tested data into two parts. The first part is modeling data used to establish a fuzzy model. The other part is validation data which is used to validate the precision of model.

(2) According to the modeling data, determine the input and output variables, the corresponding domain interval, the number of fuzzy subset, the quantification factors, and the membership functions.

(3) Quantify the modeling data, calculate the membership degree of every data in every subset, and the maximum membership degree determines the data belonging to a subset.

(4) Correlation matrix is made up of the subsets of input and output variables. Every element in the matrix denotes the appearance number of the corresponding fuzzy rule.

(5) According to the correlation matrix, write out the fuzzy rules. The modeling data are used to validate the model, and then fuzzy rules are adjusted.

(6) Validate the model using validation data, adjust the fuzzy rules. And the final fuzzy rules can be obtained.

\section{Steady-State Fuzzy Model for Speed Control of Ultrasonic Motor}

The two-input and one-output steady-state fuzzy model of ultrasonic motor system is worked out. According to the 


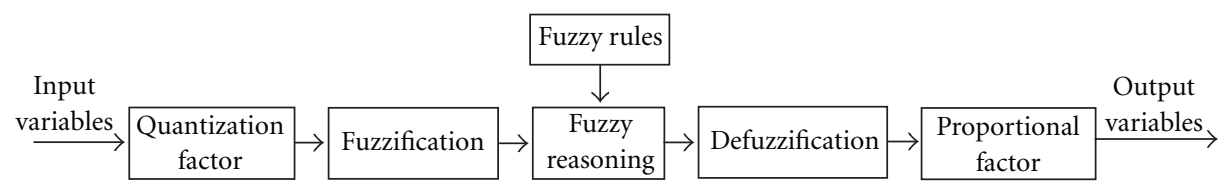

Figure 3: Basic structure of fuzzy model.

TABle 1: Membership functions of $F$.

\begin{tabular}{lccc}
\hline Fuzzy subsets & Starting points & Acmes & Ending points \\
\hline$A_{1}$ & 0 & 1 & 2.63 \\
$A_{2}$ & 2.5 & 3 & 3.1 \\
$A_{3}$ & 2.92 & 3.2 & 3.3 \\
$A_{4}$ & 3.06 & 3.4 & 3.47 \\
$A_{5}$ & 3.27 & 3.5 & 3.58 \\
$A_{6}$ & 3.31 & 3.6 & 3.72 \\
$A_{7}$ & 3.56 & 3.7 & 3.92 \\
$A_{8}$ & 3.9 & 4.5 & 4.55 \\
$A_{9}$ & 4.47 & 4.6 & 4.71 \\
$A_{10}$ & 4.68 & 5 & 5.2 \\
$A_{11}$ & 5.13 & 5.2 & 6.5 \\
$A_{12}$ & 6.36 & 7 & 8 \\
\hline
\end{tabular}

tested data, input variables contain the frequency control word $f$ and driving voltage amplitude $u$. Output variable is the rotating speed value $n$. Among the tested data, the modeling data are $n=10,30,40,70,80,90,110,120 \mathrm{r} / \mathrm{min}$, and the validation data are $n=20,100 \mathrm{r} / \mathrm{min}$. After the modeling, validation calculation can be implemented by using a fuzzy logic toolbox of MATLAB. Inputting the value of $f$ and $u$, the model output value can be obtained.

Considering the precision of tested data and model, the domain interval of two input variables is $[1,7]$, and output variables is $[0,13]$. The numbers of the subset of input variables $f, u, n$ are $12,7,6$, respectively. According to the proportional relation between the range of tested data and domain interval, the quantification factors of variables $f$, $u, n$ are set to $G f=550, G u=55, G n=10$. The quantified variables are defined as $U, F$, and $N$, respectively. The method of defuzzification is center of gravity.

Determine all the membership functions of variables. Triangle and Gaussian membership functions are often used. Triangle membership function is a piecewise linear function, and the shape is only relevant to slope. The range of application is wide, and the amount of online calculation is small. The characteristics of Gaussian membership functions are continuous graph and differential coefficient everywhere. Gaussian membership function is often used to describe the nonlinear system. Because of the nonlinearity of ultrasonic motor, the membership functions of input variables $F, U$ are triangle membership functions, and output variable $N$ is Gaussian membership functions.

After the shape is selected, membership function of every fuzzy subset is determined in the corresponding domain interval. This work is an important tache. It decides the
TABle 2: Membership functions of $U$.

\begin{tabular}{lccc}
\hline Fuzzy subsets & Starting points & Acmes & Ending points \\
\hline$B_{1}$ & 0 & 1 & 1.26 \\
$B_{2}$ & 1 & 1.9 & 2 \\
$B_{3}$ & 1.47 & 3.01 & 3.33 \\
$B_{4}$ & 2.21 & 3.97 & 4.1 \\
$B_{5}$ & 3.81 & 5.1 & 5.76 \\
$B_{6}$ & 5.45 & 5.96 & 6.82 \\
$B_{7}$ & 6.55 & 7 & 8.5 \\
\hline
\end{tabular}

TABle 3: Membership functions of $N$.

\begin{tabular}{lcc}
\hline Fuzzy subsets & Widths & Centers \\
\hline$C_{1}$ & 0.53 & 1.01 \\
$C_{2}$ & 0.959 & 3.83 \\
$C_{3}$ & 0.922 & 5 \\
$C_{4}$ & 1.21 & 6.92 \\
$C_{5}$ & 1.12 & 9.21 \\
$C_{6}$ & 1.05 & 11.8 \\
\hline
\end{tabular}

membership degree of input and output variables and can reflect the nonlinearity of object. Figure 2 shows the nonlinear relationship among the amplitude of driving voltage, frequency, and rotating speed. The same changes of driving voltage amplitude and frequency can bring on different rotating speed changes in different numerical value range. Because the density of tested data is different, the distribution of membership functions should consider the status. Freedom degree of fuzzy modeling should be fully used, and nonlinear modeling is realized by simple form. According to the principle above, after validation and adjustment, the membership functions of input and output variables are shown in Tables 1,2, and 3, respectively. The number of fuzzy subsets of variables $F, U$, and $N$ is 12,7 , and 6 , respectively. In order to explain the meanings of data in tables, the corresponding membership functions in Table 2 are show in Figure 4.

The modeling data are quantified using the decided quantification and proportion factors, and the value of input and output variables can be obtained in corresponding domain interval. Membership degree is calculated in every subset, and a subset to which the data belong is determined by the maximum of membership degree. The corresponding fuzzy relationship of input and output can be obtained, that is, fuzzy rules. According to all the fuzzy rules, correlation 
TABLE 4: Initial fuzzy rules.

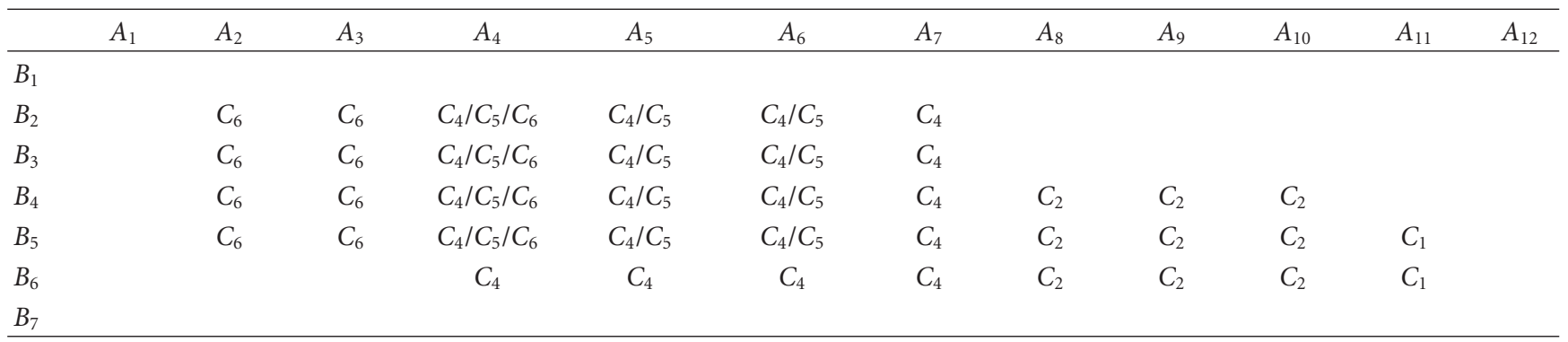

matrixes are shown in (1). Every element represents the appearance number of fuzzy rule

$$
\begin{aligned}
R_{1} & =\left[\begin{array}{ccccccc}
C_{1} & C_{2} & C_{3} & C_{4} & C_{5} & C_{6} & \\
0 & 0 & 0 & 0 & 0 & 0 & B_{1} \\
0 & 0 & 0 & 12 & 4 & 16 & B_{2} \\
0 & 0 & 0 & 26 & 13 & 32 & B_{3} \\
0 & 9 & 0 & 20 & 10 & 20 & B_{4} \\
14 & 31 & 0 & 33 & 14 & 19 & B_{5} \\
11 & 13 & 0 & 1 & 0 & 0 & B_{6} \\
0 & 0 & 0 & 0 & 0 & 0 & B_{7}
\end{array}\right], \\
R_{2} & =\left[\begin{array}{cccccccc}
C_{1} & C_{2} & C_{3} & C_{4} & C_{5} & C_{6} & \\
0 & 0 & 0 & 0 & 0 & 0 & A_{1} \\
0 & 0 & 0 & 0 & 0 & 40 & A_{2} \\
0 & 0 & 0 & 0 & 0 & 38 & A_{3} \\
0 & 0 & 0 & 7 & 14 & 3 & A_{4} \\
0 & 0 & 0 & 13 & 15 & 0 & A_{5} \\
0 & 0 & 0 & 27 & 12 & 0 & A_{6} \\
0 & 0 & 0 & 45 & 0 & 0 & A_{7} \\
0 & 34 & 0 & 0 & 0 & 0 & A_{8} \\
0 & 11 & 0 & 0 & 0 & 0 & A_{9} \\
0 & 8 & 0 & 0 & 0 & 0 & A_{10} \\
25 & 0 & 0 & 0 & 0 & 0 & A_{11} \\
0 & 0 & 0 & 0 & 0 & 0 & A_{12}
\end{array}\right] .
\end{aligned}
$$

Based on the correlation matrixes, the appearance number of fuzzy rules is large, and the rules are selected. The initial fuzzy rules are shown in Table 4. In Table 4, the first row represents 12 fuzzy subsets of input variables $F$, and the first column 7 fuzzy subsets of $U$, which denotes the premise part of fuzzy rules.

Some rules have the same premise condition and different conclusion. When the appearance number of these rules is large and close, the rules should be held in the initial fuzzy rules. In Table 4, the rules which have the same premise condition and different conclusion must be selected

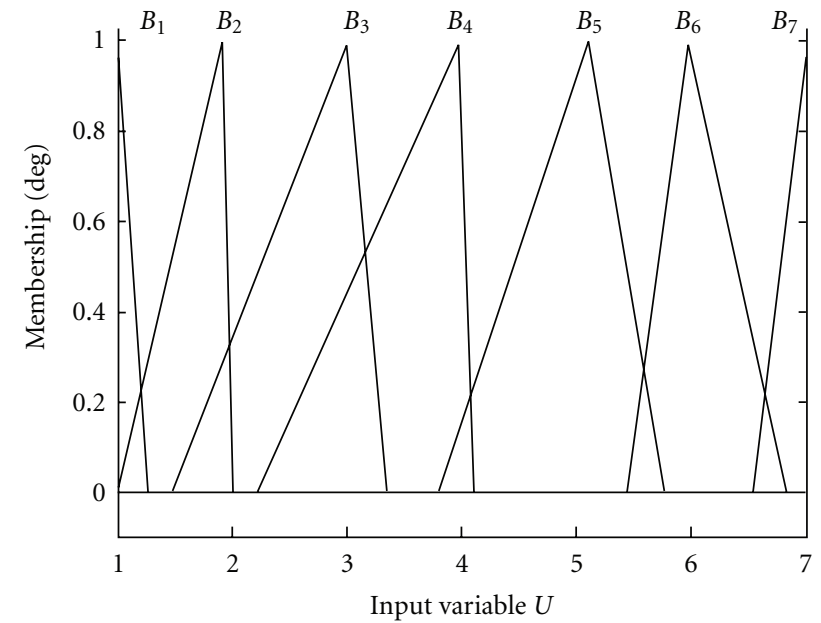

FIgURE 4: Membership functions of $U$.

by model validation. The method is as follows. Hold, add and delete rules, and save the more perfect conclusion. Variance, absolute error, and relative error of validation data are smaller in the conclusion. After being selected, the fuzzy rules are shown in Table 5.

On one hand, the fuzzy rules in Tables 4 and 5 are used to design a fuzzy model. Comparison of variance, absolute error, and relative error between Tables 4 and 5 is shown in Table 6. After rules adjustment, the precision of model the is increased. As shown in Table 6, the even value of error's variance decreases from 21.2675 to 6.4125 , and the even value of the maximum of absolute error decreases from $11.0572 \mathrm{r} / \mathrm{min}$ to $5.5502 \mathrm{r} / \mathrm{min}$.

The fuzzy model can be used to analyze the steadystate characteristic of ultrasonic motor. On the other hand, performance of speed control can be improved with the help of the fuzzy model. For example, in the speed control system with efficiency optimum control, the driving frequency of ultrasonic motor is used as control variable to adjust motor's rotating speed, and the amplitude of driving voltage is modulated to achieve optimum efficiency. Any change of voltage amplitude will lead to the change of rotating speed. Therefore, the two control variables, the amplitude of driving voltage, and driving frequency must be changed synchronously to maintain the control performance of speed. Because of the lack of an accurate theoretical model, this synchronous change of the two variables cannot be simply 
Table 5: Fuzzy rules.

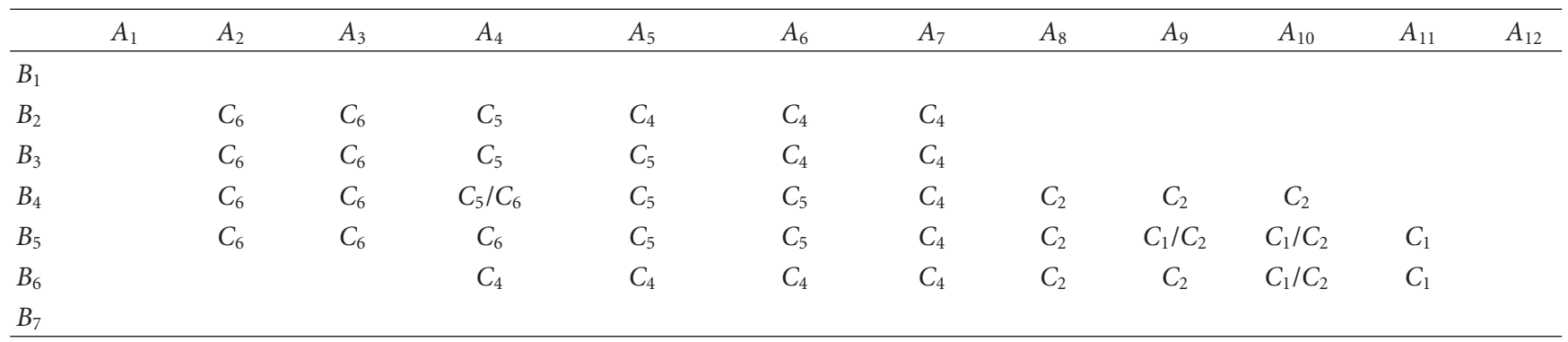

TABLE 6: Error comparison.

\begin{tabular}{lcccccc}
\hline \multirow{2}{*}{$\begin{array}{l}\text { Rotating speed } \\
(\mathrm{r} / \mathrm{min})\end{array}$} & \multicolumn{2}{c}{ Variance } & \multicolumn{2}{c}{ Absolute error $(\mathrm{r} / \mathrm{min})$} & \multicolumn{2}{c}{ Relative error $(\%)$} \\
& Table 4 & Table 5 & Table 4 & Table 5 & Table 4 & Table 5 \\
\hline 10 & 0.0113 & 0.0113 & $0.4061 \sim 0.8070$ & $0.4061 \sim 0.8070$ & $4.06 \sim 8.07$ & $4.06 \sim 8.07$ \\
30 & $4.7240 \mathrm{e}-006$ & 0.7593 & $8.2984 \sim 8.3074$ & $-1.1788 \sim 1.3960$ & $27.66 \sim 27.69$ & $0.15 \sim 3.93$ \\
40 & $2.1821 \mathrm{e}-006$ & 0.0341 & $-1.7009 \sim-1.6950$ & $-2.7430 \sim-1.6950$ & $4.24 \sim 4.25$ & $4.24 \sim 6.86$ \\
70 & 23.0633 & 4.9605 & $-0.8111 \sim 9.9208$ & $-0.8111 \sim 6.1327$ & $0.85 \sim 14.17$ & $0.65 \sim 8.76$ \\
80 & 19.2751 & 14.6837 & $-4.9175 \sim 10.0652$ & $-4.9175 \sim 12.0837$ & $0.08 \sim 12.58$ & $0.06 \sim 15.1$ \\
90 & 20.5928 & 7.0218 & $-10.3980 \sim-0.0371$ & $-4.5897 \sim 5.0962$ & $0.04 \sim 11.55$ & $0.06 \sim 5.66$ \\
110 & 96.1105 & 16.9311 & $-23.5933 \sim 5.5337$ & $-5.5518 \sim 5.5337$ & $1.74 \sim 21.45$ & $0.13 \sim 5.05$ \\
20 & 26.6029 & 4.6330 & $1.4062 \sim 18.3085$ & $1.4062 \sim 11.0495$ & $7.03 \sim 91.54$ & $7.03 \sim 55.25$ \\
100 & 5.7518 & 8.6775 & $-16.4137 \sim-5.8038$ & $-5.0923 \sim 5.0602$ & $5.8 \sim 16.41$ & $0.06 \sim 5.09$ \\
\hline
\end{tabular}

realized. The speed response in Figure 5 shows the bad performance of speed control during the efficiency optimum process.

The inverse model of the steady-state fuzzy model proposed in this paper can be used to solve this problem. According to the current value of voltage amplitude and rotating speed, the inverse model can give out the needed value of frequency to maintain the constant speed. To compensate the transient response of the adjustment of voltage amplitude, a first-order inertial element should be added to the output of the inverse fuzzy model. Using this method, better speed response can be obtained as shown in Figure 6. The maximum of absolute error during the efficiency optimum process decreases from $3.46 \mathrm{r} / \mathrm{min}$ (Figure 5) to $1.35 \mathrm{r} / \mathrm{min}$ (Figure 6).

\section{Conclusions}

Based on the experimental data, the two-input and oneoutput steady-state fuzzy model for speed control of ultrasonic motor is worked out using fuzzy modeling method. The model can well simulate the nonlinear relationship among the amplitude of driving voltage, frequency, and rotating speed. The paper shows that utilizing the nonlinear form of fuzzy logic, nonlinear modeling is easy to implement, and the nonlinear characteristics of ultrasonic motor system are fit to be described. In the process of using fuzzy

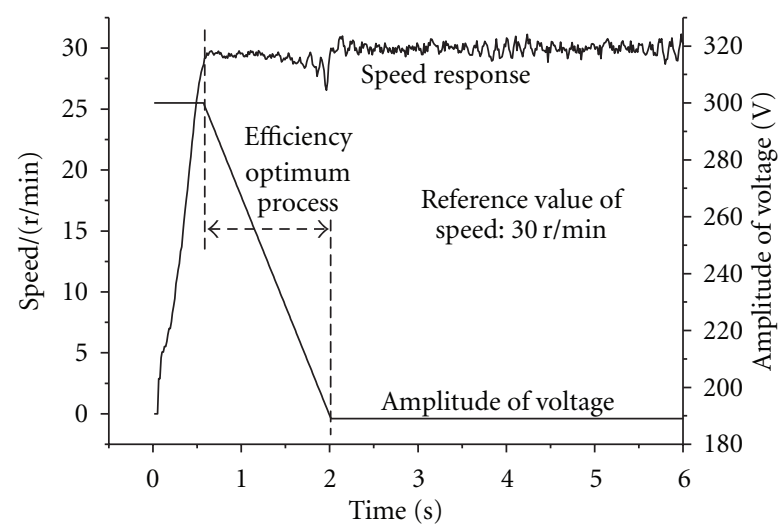

FIGURE 5: Speed response with efficiency optimum.

modeling method to establish a model, some principles can be summarized as follows.

(1) If the number of modeling data is small, modeling is relatively simple. If the number is larger, modeling is harder.

(2) When the number of quantified grade is larger in fuzzy domain interval, the effect is better. If the number is too large, the excellence of fuzzy logic is not shown, and the amount of online calculation is increased. 


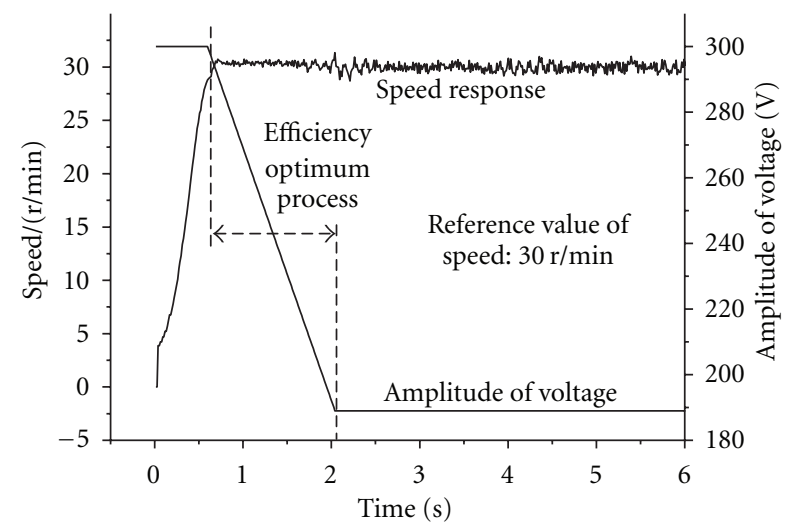

FIGURE 6: Speed response with efficiency optimum and fuzzy model.

(3) When the number of fuzzy subset is larger, description of ultrasonic motor system is more complete. With the number increasing, rules and the amount of calculation increase markedly.

(4) The span and cross range of membership functions can be decided by the distribution of tested data.

\section{Acknowledgment}

The authors are grateful for the support by the Natural Science Foundation of Henan Province through Grant no. 092300410164.

\section{References}

[1] H. Zhu, Z. Li, and C. Zhao, "An efficient approach to optimize the vibration mode of bar-type ultrasonic motors," Ultrasonics, vol. 50, no. 4-5, pp. 491-495, 2010.

[2] W. Chen, S. Shi, Y. Liu, and P. Li, "A new traveling wave ultrasonic motor using thick ring stator with nested PZT excitation," IEEE Transactions on Ultrasonics, Ferroelectrics, and Frequency Control, vol. 57, no. 5, pp. 1160-1168, 2010.

[3] B. Radi and A. El Hami, "The study of the dynamic contact in ultrasonic motor," Applied Mathematical Modelling, vol. 34, no. 12, pp. 3767-3777, 2010.

[4] T. Senjyu, M. Nakamura, N. Urasaki, H. Sekine, and T. Funabashi, "Mathematical model of ultrasonic motors for speed control," Electric Power Components and Systems, vol. 36, no. 6, pp. 637-648, 2008.

[5] N. Bigdeli and M. Haeri, "Simplified modeling and generalized predictive position control of an ultrasonic motor," ISA Transactions, vol. 44, no. 2, pp. 273-282, 2005.

[6] S. W. Chung and K. T. Chau, "Speed control of travelingwave ultrasonic motors using a practical modeling approach," Electric Power Components and Systems, vol. 35, no. 4, pp. 411428, 2007.

[7] H. Mojallali, R. Amini, R. Izadi-Zamanabadi, and A. A. Jalali, "Systematic experimental based modeling of a rotary piezoelectric ultrasonic motor," ISA Transactions, vol. 46, no. 1, pp. 31-40, 2007.

[8] S.-C. Mou and M. Ouyang, "Establishing the dynamic transfer function of a shaft-driving type ultrasonic motor by system identification," in Proceedings of the 5th World Congress on
Intelligent Control and Automation (WCICA '04), vol. 1, pp. 302-305, Seoul, Korea, Seoul, Korea.

[9] T. C. Chen and C. H. Yu, "Generalized regression neuralnetwork-based modeling approach for traveling-wave ultrasonic motors," Electric Power Components and Systems, vol. 37, no. 6, pp. 645-657, 2009.

[10] F.-J. Lin, R.-J. Wai, and C.-M. Hong, "Identification and control of rotary traveling-wave type ultrasonic motor using neural networks," IEEE Transactions on Control Systems Technology, vol. 9, no. 4, pp. 672-680, 2001.

[11] M. Jahani and H. Mojallali, "Neural network based modeling of traveling wave ultrasonic motor using genetic algorithm," in Proceedings of the 2nd International Conference on Computer and Automation Engineering (ICCAE'10), vol. 5, pp. 486-490, Singapore, February 2010. 

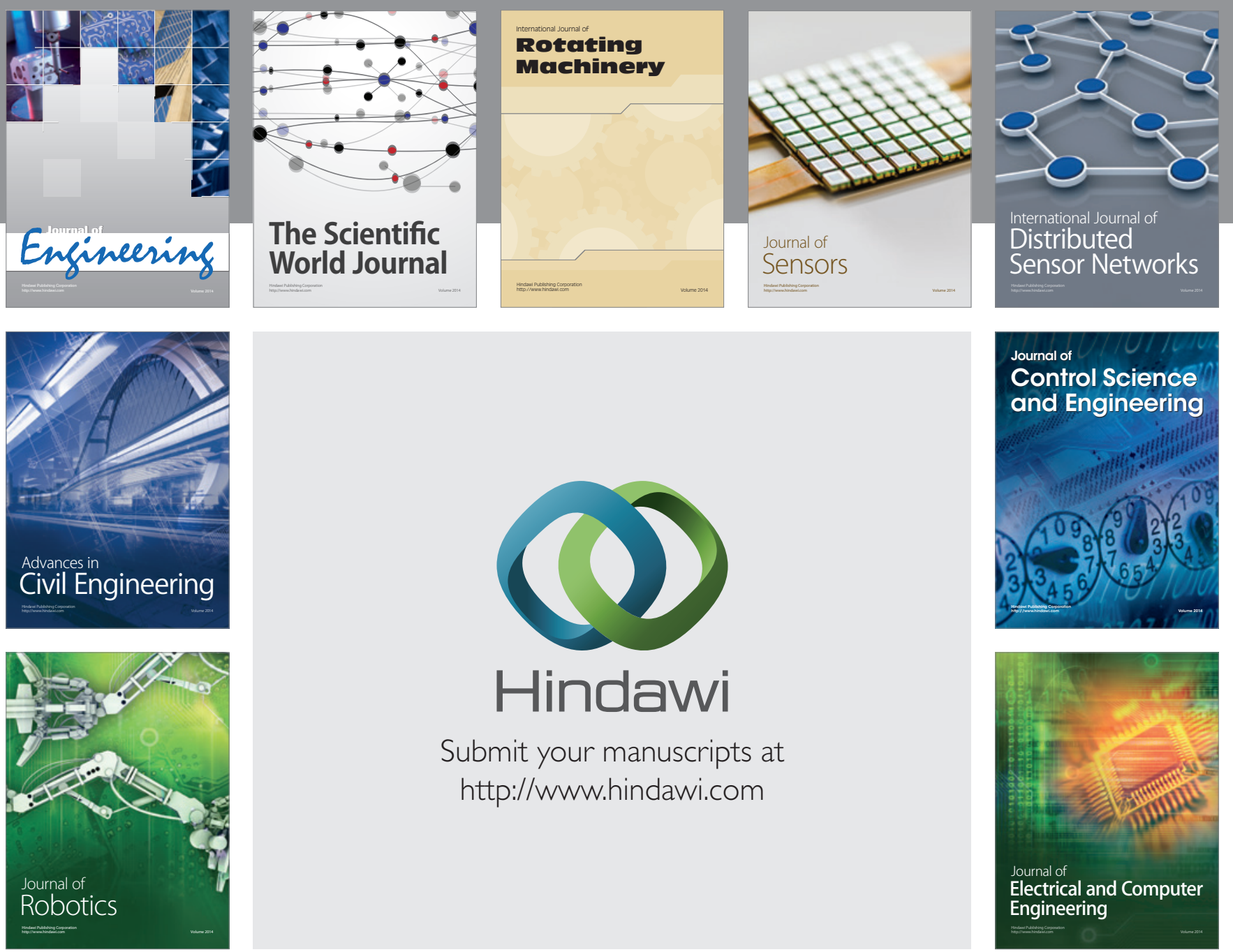

Submit your manuscripts at

http://www.hindawi.com
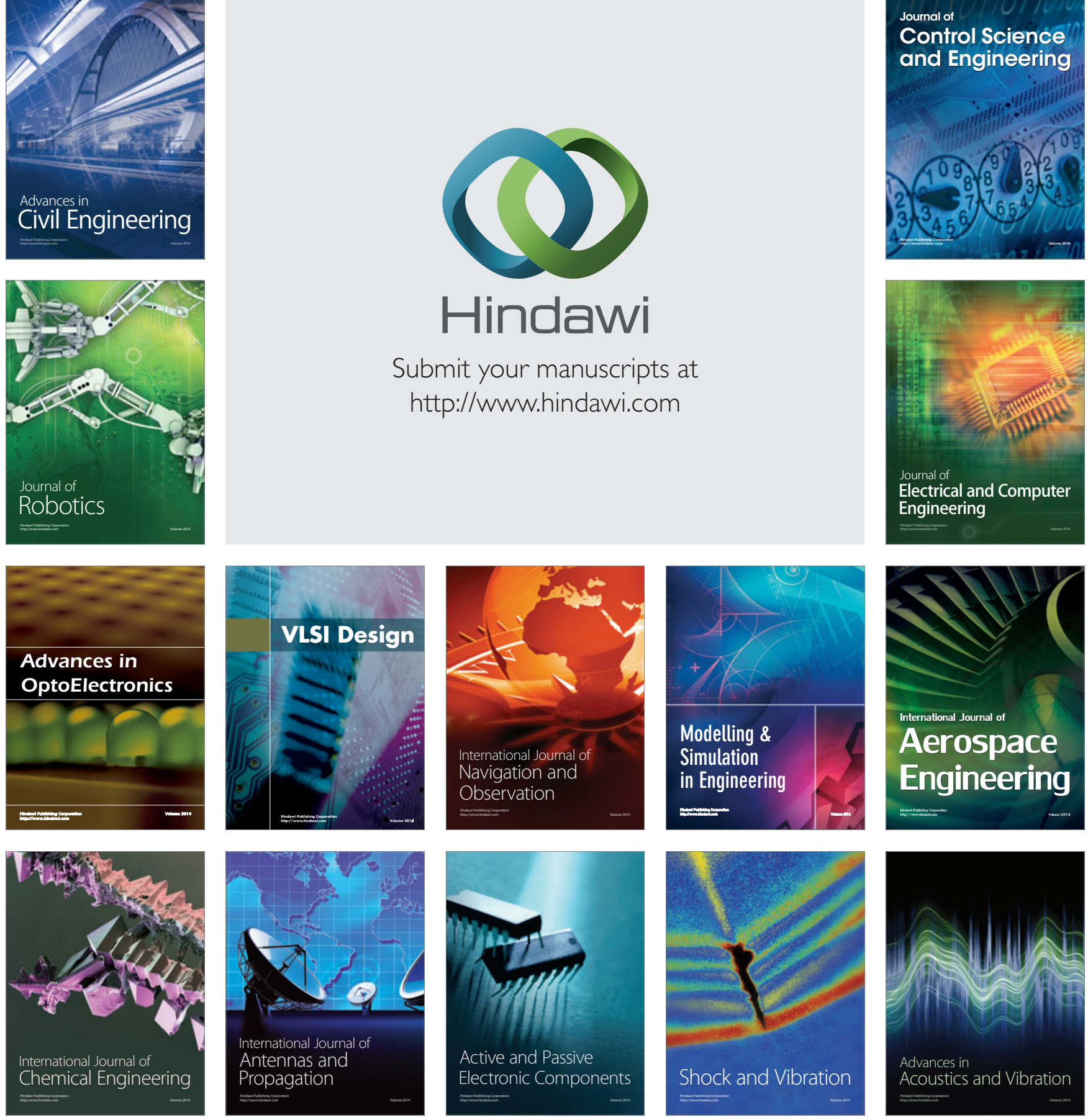